\title{
ASPECTOS MOTIVACIONAIS EM PROGRAMAS DE MUDANÇA DE COMPORTAMENTO ALIMENTAR
}

\author{
MOTIVATIONAL ASPECTS IN PROGRAMS OF NUTRITIONAL \\ BEHAVIOR CHANGES
}

\author{
Maria Alice Altenburg de ASSIS' \\ Markus Vinícius NAHAS ${ }^{2}$
}

\begin{abstract}
RESUMO
Este trabalho procurou reunir as informações concernentes às questões da adesão e motivação de indivíduos em programas de intervenção alimentar que visam à mudança do comportamento. Foram considerados os artigos publicados a partir de 1990, abordando inicialmente uma definição de termos sobre complacência, aderência, motivação, manutenção, recaída e lapso, palavras comumente utilizadas nos estudos sobre mudanças de comportamento. O tema fatores que interferem nas escolhas alimentares e na aderência à dieta trata genericamente, dos aspectos que determinam os hábitos e as preferências alimentares e tece considerações sobre as questões que permeiam a relação profissionalpaciente. A seguir foram abordadas as principais teorias motivacionais que fundamentam os programas de intervenção alimentar e algumas considerações sobre a aplicação destas teorias num programa de intervenção nutricional. O "estado da arte" dos programas de intervenção nutricional que visam à mudança do comportamento alimentar, apresentado nas publicações relatadas, aponta para a necessidade da utilização e integração dos modelos da teoria social cognitiva e treinamento profissional para aquisição de habilidades técnicas para motivar as pessoas na realização das mudanças desejáveis.
\end{abstract}

Termos de indexação: conduta na alimentação, programas de nutrição aplicada, educação nutricional, ergonomia, intervenção nutricional.

\begin{abstract}
This article is a review of the most recent publications related to the motivation and adherence factors in nutritional intervention programs directed toward behavioral changes. The review included papers published since 1990. The initial part of the article presents the definitions of several terms commonly associated with researches on the
\end{abstract}

\footnotetext{
(1) Departamento de Nutrição, Universidade Federal de Santa Catarina, Campus Universitário, Trindade, 88040-900, Florianópolis, SC.

(2) Departamento de Pós-Graduação em Educação Física e de Engenharia de Produção, Universidade Federal de Santa Catarina.
} 
behavioral change area, such as adherence, complacence, motivation, maintenance and relapse. Afterwards, the authors present information related to the factors that have been found to interfere in food choices and those that may determine the nutricional habits. The patient - professional relationship is also analysed. The following section reviews the main theories of motivation, which justify the intervention programs designed to change nutritional behavior. There seems to be a trend in the literature toward nutritional intervention programs that integrate the Social Cognitive Theorymodel and the professional training to acquire the needed skills to motivate people to promote the desired behavioral changes.

Index terms: feeding behavior, applied nutrition programmes, nutrition programmes, nutrition education, ergonomics, nutrition intervention.

\section{INTRODUÇÃO}

$\mathrm{Na}$ Europa e nos EUA, metade das mortes prematuras, em homens e mulheres com menos de 65 anos, resulta de doenças crônicas. São as doenças coronarianas, vários tipos de cânceres, anemia, bócio, cirrose do fígado, diabetes, obesidade, hipertensão arterial e osteoporose na velhice (World..., 1990).

No Brasil, o quadro não é diferente, sendo que o grupo de doenças cardiovasculares constitui a primeira causa de mortalidade (Brasil..., 1993).

Uma alimentação bem variada e balanceada, a prática regular de exercícios físicos, o controle do estresse, a adoção de um comportamento preventivo e o hábito de não usar drogas, são componentes da categoria estilo de vida, que podem ser modificados para viver melhor, com qualidade (Nahas, 1996).

Entretanto, estas mudanças no estilo de vida para a prevenção e o tratamento das doenças crônicas são caracterizadas pela baixa adesão do paciente.

Revisando a literatura sobre a falta de adesão de pacientes e os processos sociais e psicológicos que permeiam a relação médico paciente, DiMatteo (1994) relatou que $38 \%$ dos pacientes deixam de seguir um tratamento agudo recomendado (por exemplo, uso de antibióticos); $43 \%$ dos pacientes não aderem a um tratamento crônico (por exemplo, tratamento antihipertensivo), $75 \%$ dos pacientes não seguem as recomendações médicas relacionadas as mudanças no estilo de vida, como restrições alimentares, abandono do fumo e outros.

Relativo à adesão em programas de exercícios físicos, as pesquisas têm documentado que $50 \%$ dos indivíduos que se iniciam em um programa dessa natureza o abandonarão dentro de três a seis meses (Dishman, 1995).

No Serviço de Nutrição Clínica Ambulatorial do Hospital Universitário da Universidade Federal de Santa Catarina, entre 1985 e 1988, Carmo \& Batista (1994), explicitaram sua preocupação com a adesão do paciente ao tratamento dietoterápico prescrito, indicando que dos 472 pacientes atendidos, 50,6\% abandonaram o tratamento.

As pessoas que persistem numa dieta de valor energético muito baixo, podem perdem até $20 \mathrm{~kg}$ em média em 12 a 16 semanas. A maioria destes indivíduos, contudo, recuperam o peso dentro de um curto período de tempo. Muitos deles então reiniciam o processo de dieta e recomeçam o ciclo (Williams et al., 1996).

Este trabalho procurou identificar o "estado da arte" dos programas de intervenção nutricional que visam a mudança desejável do comportamento alimentar, coletando informações de publicações a partir de 1990, considerando:

- os fatores interferentes nas escolhas alimentares e na motivação e aderência dos pacientes às prescrições dietéticas;

- os tipos de programas, estratégias de intervenção e resultados obtidos,

- as teorias e principais modelos utilizados nos programas de intervenção nutricional.

Parte-se da hipótese de que os programas atualmente consideram, em geral, que os indivíduos estão prontos para a ação (mudança de comportamento), pressuposto que tem se mostrado insustentável na maioria das situações. 
Definição de Termos

Apesar dos termos complacência e adesão serem utilizados invariavelmente na literatura, dando a impressão que têm o mesmo significado de adesão, para Holli \& Calabrese (1991) eles apresentam uma diferenciação:

- Complacência tem sido definida como a extensão na qual o comportamento alimentar e os alimentos consumidos pelo paciente coincidem com as recomendações e prescrições dietéticas. Neste caso, o "conselheiro" é quem decide autoritariamente o que seria melhor para o indivíduo, que é passivo e complacente.

- A palavra adesão pode sugerir maior participação do cliente na resolução dos problemas e tomada de decisões sobre as mudanças alimentares, que são comportamentos voluntários.

A motivação pode ser conceituada como alguma coisa que faz uma pessoa agir, ou o processo de estimular uma pessoa a agir. A palavraéfreqüentemente utilizada para descrever aqueles processos que instigam um comportamento; fornecem direção e propósito ao comportamento; permitem a persistência do comportamento; conduzem às escolhas ou preferências de um determinado comportamento(Holli \& Calabrese, 1991).

A motivação é complexa e muitas variáveis intrínsecas e extrínsecas, influenciam o processo em determinado momento. As influências motivacionais de hoje podem ser diferentes do amanhã, e metas a curto prazo podem preceder sobre as de longo prazo. O problema é que, tornar-se ou permanecer saudável, ou aprender o que alguém precisa para um cuidado apropriado em diabetes ou doença cardiovascular, envolve metas a longo prazo, enquanto comer uma torta de chocolate, "só desta vez", satisfaz uma meta a curto prazo de prazer (Rollnick, 1996).

A motivação intrínseca surge do indivíduo, pertence aos seus desejos, necessidades, direções ou metas. Um indivíduo que recentemente sofreu um ataque cardíaco pode estar intrinsicamente motivado a mudar sua prática alimentar. Fatores externos ou extrínsecos podem suplementar positiva ou negativamente esta motivação. Exemplos de fatores externos que atuam positivamente, incluem o suporte familiar, o prazer e as recompensas materiais. Uma motivação pessoal em seguir as recomendações dietéticas, pode ser prejudicada em ocasiões sociais, ou devido a falta de suporte familiar e de amigos (Horn et al., 1997).

No controle de peso, a manutenção pode ser definida como uma perda de peso que será mantida ao longo do tempo. Aocontrário, a recaídaé a subseqüente recuperação do peso perdido (Sternberg, 1993).

Não existe um acordo geral de como quantificar estes constructos e a literatura contém várias definições utilizadas por diferentes investigadores, citados por Sternberg (1993):

- perdedores bem sucedidos: indivíduos que perderam $9 \mathrm{~kg}$ ou mais e mantiveram a perda por um ano ou mais;

- recuperadores: indivíduos que perderam peso, mas recuperaram $20 \%$ ou mais;

- mantenedores: indivíduos que recuperaram menos de $20 \%$;

- maus mantenedores: indivíduos que recuperaram mais de $50 \%$ do peso previamente perdido;

- bons mantenedores: indivíduos que recuperaram menos de $20 \%$ do peso previamente perdido.

Uma outra definição de recaída baseia-se na suposição comum de que mudanças em comportamento alvo, como a quantidade de comida consumida, levam a perda de peso. Esses comportamentos alvo funcionam como regras regulando a ingestão. Aceitando esta definição, a manutenção poderia ser definida como o uso continuado de regras regulando o comportamento de ingesta. A recaída pode ser considerada como a violação de uma ou mais dessas regras (Sternberg, 1993).

O modelo Prevenção de Recaída(PR) cognitivo-comportamental define lapso como a primeira ocorrência de uso de substância alvo após um período de abstinência. Quando uma dieta prescrita envolve estrita adesão a um dado nível energético ou a listas de alimentos, um lapso inicial, pode ser considerado o fracasso em aderir a estas recomendações específicas. Para programas comportamentais que visam mudar os hábitos alimentares e de atividade física, sem uma prescrição de nível energético específico e uma amplitude considerável na escolha de alimentos, uma definição apropriada de um lapso inicial poderia ser mais subjetiva, como qualquer exemplo de ingestão 
planejada e não controlada, na qual o indivíduo comeu mais do que o esperado ou planejado, sem um plano compensatório anterior (Sternberg, 1993).

Fatores que interferem nas escolhas alimentares e na aderência à dieta

Dentro de uma perspectiva mais ampla, os hábitos alimentares são adquiridos em função de aspectos culturais, antropológicos, socioeconômicos e psicológicos, que envolvem o ambiente das pessoas.

A seleção de alimentos é uma parte de um sistema comportamental complexo. Na criança é determinada primeiramente pelos pais, práticas culturais e éticas de seu grupo. Experiências precoces e interação contínua com o alimento determinam as preferências alimentares, hábitos e atitudes exibidas pelos adultos. Outras influências incluem o preço do alimento, o valor do prestígio do alimento, religião, geografia, pares e influências sociais, preparação e estocagem do alimento, habilidades no preparo de alimentos, disponibilidade de tempo e conveniência, bem como as preferências e intolerâncias pessoais. Citam-se também os fatores afetivos, envolvendo atitudes, crenças e valores (Holli \& Calabrese, 1991).

As qualidades sensoriais (sabor, cheiro, textura e aparência) são fortes determinantes do comportamento alimentar. As propriedades sensoriais dos alimentos desempenham um papel não somente na determinação do seu consumo, como também na determinação da saciedade, ingestão e seleção do alimento numa refeição (Mattes \& Kare, 1994).

Citam-se também, os aspectos fisiológicos, neurofisiológicos e o papel dos neurotransmissores cerebrais envolvidos nos mecanismos de regulação da ingestão de macronutrientes e sua influência sobre a escolha dos alimentos e tipos de refeição (Anderson, 1994).

Um dos papéis do nutricionista é o de ajudar as pessoas a modificar seus hábitos alimentares, através da assistência nutricional a indivíduos e grupos populacionais (Baldwin \& Falciglia, 1995).

Assistência é mais do que fornecer uma informação. Livros, revistas, reportagens em jornais ou na televisão, família e amigos podem prover informação, sem o auxílio de profissionais. A resolução dos problemas, contudo, e a descoberta pessoal do significado das soluções para a sua vida, vêm da interação entre o profissional assistente e o indivíduo assistido, bem como da tecnologia empregada (Holli \& Calabrese, 1991).

O conhecimento sobre o que comer é um primeiro degrau na influência do comportamento alimentar saudável, provavelmente super valorizado. A relação entre o que as pessoas sabem e o que as pessoas fazem, tem sido considerado como "altamente tênue". O conhecimento não instiga a mudança, mas funciona como um instrumento quando as pessoas desejam mudar (Chapman et al., 1995).

Muitos fatores influenciam a adesão do cliente ou paciente a uma prescrição dietética, sendo geralmente relatadas e analisadas em termos de quatro conjuntos de características em relação: a) ao paciente e cliente; b) ao profissional; c) ao serviço ou clínica, e d) à prescrição (Holli \& Calabrese, 1991).

Através da Análise Ergonômica do Trabalho (AET) em um ambulatório de nutrição, Assis et al., (1996) identificaram aspectos inadequados no posto de trabalho do nutricionista relativos à ambiência (luminosidade, ruído, ventilação, mobiliário, equipamentos), à organização do ambulatório (marcação de consultas e retornos, tempo de consulta, dados de prontuário médico, tempo de espera de atendimento, falta de microcomputador) e à prescrição (tempo limitado de consulta, falta de material ilustrativo, uso de dieta padronizada). Estes condicionantes foram discutidos como interferentes na relação profissional-paciente e como possíveis causas contribuintes ao abandono dos pacientes no programa.

A satisfação com o nível do atendimento e com a atitude do profissional tem sido relatada como fatores que influenciam a aderência. Por outro lado, a aderência é melhor estabelecida se o paciente encontra o mesmo profissional em cada visita. As características do serviço institucional ou da clínica são importantes. A espera para o atendimento pode ser um fator que conduz à desistência de uma próxima visita. Um ambiente acolhedor por parte de toda a equipeé fundamental para criar um clima aconchegante (Holli \& Calabrese, 1991).

As características da prescrição têm sido citadas como um dos fatores mais importantes na adesão. Destas, a complexidade é a mais significativa. A dificuldade de adaptar o novo regime à rotina diária, a mudança no estilo de vida, a falta de acesso 
a alimentos apropriados, ou esforços extras requeridos na preparação do alimento são condicionantes impostas à prescrição (Coyne et al., 1995).

No Multiple Risk Factor Intervention Trial (MRFIT), Horn et al., (1997) encontraram uma forte associação entre as variáveis sócio-demográficas e os fatores de risco cardiovascular, coletadas no início do programa e a adesão à dieta, avaliada durante o seguimento da intervenção em 6 anos, sendo maior para os participantes mais velhos, de cor branca, não usuários de bebida alcoólica, menos estressados, que faziam as refeições fora de casa com menor freqüência, que apresentaram menor sobrepeso, com taxas de colesterol e pressão sangüínea diastólica mais elevadas e nos não fumantes.

A maioria dos programas de educação nutricional e pesquisas publicadas nesta área não citam uma teoria ou um modelo particular que fundamente a prática ou a pesquisa. Observa-se, nestas publicações, um vácuo, tanto na familiaridade com estas teorias quanto na habilidade em aplicá-las (Glanz \& Erickson, 1993).

Teorias motivacionais nos programas de mudança do comportamento alimentar

As teorias que embasam os programas de educação nutricional têm sido derivadas do modelo teórico "Teoria Social Cognitiva" (TSC).

A TSC proposta por Bandura citado por Baldwin \& Falcigia (1995) descreve o comportamento humano como sendo reciprocamente determinado pelas disposições internas e influências ambientais. O comportamento, fatores pessoais internos (cognitivos) e os eventos ambientais interagem entre si bidirecionalmente.

Recentemente, a TSC tem sido utilizada em conjunto com a psicologia cognitiva para formar a "Teoria Cognitivo-Comportamental" (Baldwin \& Falcigia , 1995).

Na psicologia cognitiva, os métodos são dirigidos para modificações de sentimentos e ações, influenciando um padrão de pensamento social. Estratégias são dirigidas à mudança de atitudes individuais, crenças e percepções sobre o comportamento (Prochaska \& DiClemente 1986).

A teoria social cognitiva e a psicologia cognitiva podem ser utilizadas para conduzir o processo de mudança cognitiva. A mudança de comportamento individual pode ser facilitada pela modificação dos fatores pessoais internos. Estes fatores incluem não somente a obtenção dos conhecimentos necessários e práticas apropriadas para fazer uma mudança, mas também um componente de auto desenvolvimento. Apesar de uma pessoa ter o desejo de realizar uma mudança e seja definitivamente a responsável pela modificação do comportamento alimentar, o nutricionista poderá ajudá-la no desenvolvimento pessoal de auto-eficácia, autocontrole e auto-avaliação, três aspectos críticos da mudança (Baldwin \& Falciglia, 1995).

Collins citado por Sternberg (1993) comparou as abordagens dos tratamentos cognitivo, comportamental e cognitivo-comportamental combinado em um programa de redução de peso de oito semanas. No grupo de terapia cognitiva, os participantes foram ensinados a reconhecer e a mudar pensamentos negativos e auto-relatos a respeito da comida, do seu peso e dos esforços para perder peso. As técnicas incluíam reestruturação cognitiva, treinamento auto-instrucional e terapia racional emotiva. Após um acompanhamento de sete meses, os resultados mostraram que o grupo de tratamento comportamental perdeu mais peso que o grupo controle. A diferença entre os grupos cognitivo-comportamental e controle foi significativa, enquanto o grupo cognitivo não diferiu do grupo controle na quantidade de peso perdida. Durante os três primeiros meses de manutenção, participantes em todos os quatro grupos continuaram a perder peso, com a maior média de perda ocorrendo no grupo cognitivo-comportamental.

\section{Treinamento do auto controle comportamental}

O treinamento do autocontrole comportamental tem sido a base teórica da maioria dos programas de tratamento da obesidade moderada. Este modelo de tratamento acredita que a obesidade é resultado de um excesso de ingestão energética e de uma insuficiente atividade física. Também assume que os indivíduos podem ser treinados a usar métodos de autocontrole para efetuar as mudanças de comportamento relativas à ingestão alimentar e à prática de exercícios. A curto prazo, os participantes de tais programas fazem as mudanças apropriadas e perdem peso. Contudo a maioria dessas pessoas experimentam um sucesso temporário na manutenção do peso perdido. Em muitos 
indivíduos, os relapsos estão relacionados ao desenvolvimento de ingestão compulsiva, caracterizada pela falta de controle de ingesta e dependência alimentar (Goodrick \& Foreyt, 1991).

Segundo Marlatt \& Gordon (1993), a essência do modelo de autocontrole é que o indivíduo move-se de uma posição de ser o "cliente", sob a orientação de um terapeuta, para uma posição na qual se torna mais capaz de assumir a responsabilidade pelo processo de mudança.

Williams et al. (1996), testaram durante seis meses a teoria do autocontrole em 128 pacientes com obesidade mórbida ou severa, participantes de um programa de perda de peso com dietas de valor energético muito baixo. O programa tinha a duração de 23 meses. As variáveis ambientais e psicológicas, relacionadas à motivação do paciente, foram usadas para predizer: a) participação semanal dos pacientes no programa durante 6 meses, b) a perda de peso durante este período e c) o exercício e a manutenção da perda de peso no seguimento de 23 meses. As análises confirmaram o prognóstico de que, os participantes cuja motivação para a perda de peso era autônoma freqüentavam com mais regularidade o programa, perdiam mais peso e apresentavam maior manutenção da perda de peso durante o seguimento. Além disso, a motivação autônoma do participante para perder peso poderia ser prognosticada pela sua orientação e suporte autônomos percebidos no clima interpessoal criado pelo pessoal de saúde.

\section{Health Belief Model}

O Health Belief Model (HBM) formulado por Becker \& Mainan, em 1975, (Miranda Ramos, 1996) é postulado como uma probabilidade de adoção ao comportamento apropriado para a prevenção e controle de algumas doenças, dependendo da percepção do indivíduo de uma ameaça a saúde pessoal, e a convicção de que a ação recomendada reduziria esta ameaça. $\mathrm{O}$ modelo propõe que um conjunto de crenças contribuam para a motivação e engajamento a um comportamento particular relacionado a saúde (Chapman et al., 1995).

Williams (1996) relata que o HBM tem sido a abordagem motivacional mais freqüentemente aplicada nos programas de saúde. Recentes formulações do modelo, incorporaram os conceitos do "locus de controle" e da auto-eficácia (Williams et al., 1996). Quando aplicada na perda de peso, esta teoria sugere que as pessoas poderão ser motivadas, se elas acreditarem que a perda de peso diminuirá a probabilidade de contrair uma doença que ameace suas vidas; se elas possuírem um "locus de controle interno" e esperarem que comportamentos específicos, tais como a redução da ingestão energética e a prática de exercícios, resultarão em significativa perda de peso e se elas estiverem confiantes na sua capacidade de efetuar os comportamentos necessários.

\section{Modelo de Prevenção de Recaída}

O modelo Prevenção de Recaída (PR) cognitivo-comportamental (Marlatt \& Gordon, 1993), baseia-se no constructo de auto-eficácia e o utiliza como um conceito explanatório, na compreensão de como se espera que uma violação inicial de regras seja capaz de influenciar o posterior uso de substâncias e a recaída.

A maioria dos programas de redução de peso menciona pouco a possibilidade de um lapso ou uma recaída, ou a maneira de enfrentar algum deles, se ocorrer. Isto efetivamente priva os indivíduos de desenvolver habilidades para enfrentar essas situações e/ou minimizar o dano se alguma delas ocorrer.

O modelo PR pode ser usado para examinar processos de recaída e manutenção da perda de peso (Sternber, 1993).

\section{Modelo Transteorético}

O modelo Transteorético (MT) (Prochaska et al., 1992), também chamado de teoria de estágios de mudança, descreve a mudança de comportamento como um processo no qual os indivíduos progridem, através de uma série de fases discretas ou estágios de mudança. Estes estágios de mudança têm sido documentados na mudança comportamental de indivíduos, nas áreas de cessação do fumo, uso de substâncias, controle do peso, uso de protetor solar, uso de preservativos e promoção da atividade física.

Os cinco estágios: pré-contemplação, contemplação, preparação, ação e manutenção são descritos em Maibach \& Cotton (1995) como:

1. A Pré-contemplação é o estágio no qual um indivíduo não tem intenção de mudar um comportamento relevante num futuro previsto. Neste estágio, as pessoas não percebem ou recusam o conhecimento do risco, ou ainda decidiram por alguma razão não adotar um comportamento mais saudável. 
2. contemplação é o estágio no qual um indivíduo começa a considerar a necessidade de mudar o comportamento em algum ponto do futuro. As pessoas freqüentemente permanecem neste estágio por um longo período de tempo devido à dificuldade de avaliação dos custos e benefícios da mudança de seu comportamento.

3. No estágio da preparação, o indivíduo toma a decisão de mudar o seu comportamento, sendo mais comumente caracterizado como um período de planejamento da estratégia da mudança de comportamento.

4. Ação é o estágio no qual, os indivíduos implementam o seu plano de mudança do comportamento e começam a efetuá-lo de uma maneira consistente.

5. A manutenção é o estágio final, no qual a prática comportamental já está solidificada e incorporada na rotina, sendo caracterizada por esforços para prevenir relapsos. O relapso pode ocorrer em qualquer parte desta sequiência, podendo ou não ser seguido por uma interrupção do progresso através dos estágios de mudança.

Os estágios de mudança fornecem uma descrição de quando ocorrem trocas particulares em atitudes, intenções e comportamentos.

Um segundo componente do modelo Transteorético, os processos de mudança, descrevem como estas trocas ocorrem nos diversos estágios. Processos de mudança são atividades e experiências de enfrentamento, encobertas e aparentes, que os indivíduos se engajam na tentativa de modificar o comportamento. Um aspecto importante acerca dos processos de mudança é o de que cada processo é mais influenciável em certos estágios do que em outros. As pessoas passam eficientemente através dos estágios quando processos apropriados a cada um deles são utilizados. Se alguns processos são usados excessivamente, em estágios inapropriados, eles podem obstruir a mudança ou precipitar um relapso num estágio precoce (Prochaska et al., 1992).

Integração dos modelos teóricos nos programas de intervenção visando mudança do comportamento alimentar

Glanz \& Erickson (1993) descreveram a aplicação de quatro modelos teóricos numa intervenção nutricional para reduzir os riscos de doenças crônicas entre trabalhadores de usinas energéticas rurais. O projeto é um estudo de campo prospectivo em 40 locais de trabalho com mais de 7 mil trabalhadores. É componente do projeto Working Well, implementado pelo National Institute of Cancer dos EUA, para testar a efetividade de intervenções de promoção à saúde no local de trabalho, visando mudanças organizacionais e individuais para diminuir o risco de câncer.

Os modelos teóricos utilizados foram: 1) Processamento de informações do consumidor; 2) Estágios de mudança (Modelo transteorético); 3) Teoria social cognitiva, 4) Difusão de inovações.

Os três primeiros modelos, processamento de informações ao consumidor, estágios de mudança e teoria social cognitiva, tratam do papel dos pensamentos e julgamentos, denominadas cognições, e como estas cognições influenciam o comportamento de saúde. O quarto modelo, difusão de inovações, está relacionado ao modo como os educadores nutricionais podem mais efetivamente propagar a adoção de novos e saudáveis hábitos alimentares, dentro de uma comunidade. Os modelos foram integrados num plano de intervenção que focaliza os níveis organizacional, ambiental e individual.

\section{CONSIDERAÇÕES FINAIS}

O "estado da arte" dos programas de intervenção nutricional que visam a mudança do comportamento alimentar, apresentado nas publicações relatadas, aponta para a necessidade da utilização e integração dos modelos da teoria social cognitiva e treinamento profissional para aquisição de habilidades técnicas para motivar as pessoas na realização das mudanças desejáveis.

Uma abordagem fundamentada na teoria pode guiar o desenvolvimento do programa e prover uma fundamentação para a avaliação do impacto e a identificação de pontos fracos.

Os aspectos que influenciam a adesão de um paciente a uma prescrição dietética e sua motivação para adotar um padrão desejável de comportamento alimentar, devem ser analisados como um conjunto de características relacionadas ao profissional, paciente, qualidade da relação profissional-paciente, prescrição, 
aspectos organizacionais, pessoais, ambientais e físicos do serviço ou clínica e ambiente externo ao serviço.

A perspectiva da teoria social cognitiva na mudança de comportamento em relação a saúde, é a de que esta mudança pode ser facilitada pela modificação dos fatores pessoais internos (cognições) e pela alteração dos fatores ambientais para promover um comportamento mais saudável. Apesar de ambas as opções serem viáveis, os programas e campanhas de promoção a saúde, geralmente tentam influenciar mais os fatores pessoais do que os ambientais (Maibach \& Cotton, 1995).

Considerando esta teoria, a hipótese é a de que um programa de intervenção nutricional pode ser bem sucedido, se estiver embutido numa perspectiva ecológica de promoção a saúde, enfocando-se os fatores ambientais, organizacionais e pessoais que influenciam a mudança do comportamento alimentar.

A utilização das abordagens ergonômica e antropotecnológica, através das suas metodologias específicas, podem ser um poderoso instrumento nestes programas institucionais, uma vez que são orientadas para o trabalho, para as atividades daqueles que as produzem. A partir dos resultados da análise do trabalho, pode-se conceber uma árvore de causas que permite assinalar as anomalias críticas do processo de trabalho. Na ergonomia, esta árvore de causas é representada pela situação de trabalho. A antropotecnologia que é a adaptação da tecnologia ao local de transferência, permite o entendimento do sistema de trabalho e do ambiente em que o mesmo se encontra, considerando a influência dos fatores geográficos, demográficos, econômicos, epidemiológicos, sociológicos e antropológicos (Proença, 1996).

Considerando que os programas de intervenção nutricional e as teorias que os fundamentam, devem ser adaptados as características locais para atingirem as necessidades específicas da população, a identificação desses fatores pode ser proveitosa ao desenvolvimento de estratégias que influenciam a mudança comportamental.

Se, por um lado, a educação nutricional depende do uso das teorias e modelos de motivação apropriados para conduzir à prática numa dada situação, por outro, só o treinamento de habilidades das técnicas cognitivas não seria suficiente. É preciso integrar os modelos para aplicá-los no contexto organizacional, ambiental e pessoal.

\section{REFERÊNCIAS BIBLIOGRÁFICAS}

ANDERSON, G.H. Regulation of food intake. In: SHILS, M.E., OLSON, J.A., SHIKE, M. Modern nutrition in health and disease. 8.ed. Pensylvania : Lea \& Febiger, 1994. v.1: p.524-536.

ASSIS, M.A A., LAGOS THÉ, M.A., GONTIJO, L.A Análise ergonômica do posto de trabalho do nutricionista: ambulatório de nutrição clínica do Hospital Universitário. In: CONGRESSO LATINO- AMERICANO DE ERGONOMIA, 4., CONGRESSO BRASILEIRO DE ERGONOMIA, 8, 1988, Florianópolis. Anais... Florianópolis, 1998. p.1073-1078.

BALDWIN, T., FALCIGLIA, G.A. Application of cognitive behavioral theories to dietary change in clients. Journal of the American Dietetic Association, Chicago, v.95, n.11, p.1315-1317, 1995.

BRASIL. Ministério da Saúde. Coordenação de doenças cardiovasculares. Doenças cardiovasculares no Brasil. Sistema Único de Saúde - SUS. Brasília, 1993. 36p.

CARMO, M.G.T.C., BATISTA, S.M. Experiência no atendimento ambulatorial em ambulatório. Revista de Ciências da Saúde, Florianópolis, v.12, n.1/2, p.95107, 1994.

CHAPMAN, K. M., HAM, J.O., LIESEN, P., WINTER, L. Appeying behavioral models to dietary education of eldery diabetic patients. Journal of Nutrition Education, Berkeley, v.27, n.2, p.75-79, 1995.

COYNE, T., OLSON, M., BRADHAM, K., GARCON, M., GREGORY, P., SCERCH, L. Dietary satisfaction correlated with adherence in the modification of diet in renal disease study. Journal of the American Dietetic Association, Chicago, v.95, n.11, p.1301-1306, 1995.

DiMATTEO, M.R. Enhancing patient adherence to medical recommendations. Journal of the American Medical Association, Chicago, v.271, n.1, p.79-83, 1994.

DISHMAN, R. K. Advances in exercise adherence. Champaign : Human Kinetics, 1995. 406p.

GOODRICK, G.K., FOREYT, J.P. Why treatments for obesity don't last. Journal of the American Dietetic Association, Chicago, v.91, n.10, p.1243-1247, 1991.

GLANZ, K., ERICKSON, M.P. Individual and community models for dietary behavior change. Journal of Nutrition Education, Berkeley, v.25, n.2, p.80-86, 1993. 
HOLLI, B.B., CALABRESE, R.J. Communication and education skills: the dietitian guide. 2.ed. Pensylvania : Lea \& Febiger, 1991. p.1-18.

HORN, L.V.V., DOLECEK, T.A., GRANDITS, G.A., SKWERES, L. Adherence to dietary recommendations in the special intervention group in the Multiple Risk Factor Intervention Trial. American Journal of Clinical Nutrition, Bethesda. v.65,. p.289S-304S, 1997. Supplement.

MAIBACH, E., COTTON, D. Moving people to behavior change. In: MAILBACH, E. \& PARROT, L. Designing health messages. London : SAGE Publications, 1995. p.41-64.

MARLATT, G.A., GORDON, J.R. Prevenção de recaída. Porto Alegre : Artes Médicas, 1993. 501p.

MATTES, R.D., KARE, M.R. Nutrition and the chemical senses. In: Modern Nutrition in health and disease. 8.ed. Pensylvania : Lea \& Febiger, 1994. v.1: p.524-536.

MIRANDA RAMOS, V. Motivação para atividade física em programas de reabilitação cardíaca. Florianópolis, 1996. 76p. Monografia submetida ao Programa de Pós Graduação em Educação Física, área de Atividade Física e Saúde, como requisito parcial para obtenção do Título de Especialista. Centro de Desportos, Universidade Federal de Santa Catarina, 1996.

NAHAS, M.V. O pentágono do bem-estar. Boletim do NuPAF, Florianópolis, v.2, n.7, p.1, 1996.
PROCHASKA, J.O., DiCLEMENTE, C.C. Towards a comprehensive model of change. In: MILLER, W., HEATHER, N. (eds). Treating addictive behaviors. New York : Plenum Press, 1986. p.3-27.

PROCHASKA, J.O., NORCROSS, J.C., FOWLER, J.L., FOLLICK, M.J., ABRAMS, D.B. Attendance and outcome in a work site weight control program: processes and stages of changes as process and prediction variables. Addictive Behaviors, Oxford, v.17, p.35-45, 1992.

PROENÇA, R.P.C. Aspectos organizacionais e inovação tecnológica em processos de transferência de tecnologia: uma abordagem antropotecnológica no setor de alimentação coletiva. Florianópolis, 1996. 306p. Tese (Doutorado em Engenharia) - Universidade Federal de Santa Catarina, 1996.

ROLLNICK, S. Behavior change in practice: targeting individuals. International Journal of Obesity, London, v.20, suppl.1, p.S22-S26, 1996.

STERNBERG, B. Recaída no controle do peso: definições, processos e estratégias de prevenção. In: MARLATT, G.A., GORDON, J.R. Prevenção de recaída. Porto Alegre : Artes Médicas, 1993. p.464-486.

WILLIAMS, G.C., GROW, V.M., FREEDMAN, Z.R., RYAN, R.M., DECI, E.L. Motivational predictors of weight loss and weight loss maintenace. Journal of Personality and Social Psychology, Washington DC, v.70, n.1, p.115-126, 1996.

WORLD HEALTH ORGANIZATION. Diet, nutrition and the prevention of chronic disease. Geneve, 1990. p.57-96. (Technical Report Series, 797).

Recebido para publicação em 16 de outubro de 1997 e aceito em 5 de maio de 1998. 